\section{Habit strength, drive, and drug effects: Round 2}

\author{
R. B. PAYNE, J. C. ROOK, and R. D. PETERS \\ University of Georgia, Athens, Ga. 30601
}

The effect of chlorpromazine (CPZ) on resistance of leverpressing to extinction was examined under varying levels of habit strength $(H)$ and drive (D). Resistance was decreased by CPZ and increased by augmented $\mathrm{H}$ and $\mathrm{D}$. The increments of resistance were essentially alike for CPZ and placebo groups, in accordance with theoretical expectation.
Does the magnitude of a depressant drug effect on learned behavior $(R)$ depend on levels of habit strength $(\mathrm{H})$ and drive (D)? Payne (1958) introduced this question in Hullian terms (1943) by supposing that depressant drugs raised reaction threshold (L), thereby reducing the magnitude of superthreshold effective reaction potential $(\bar{E}>L)$. On this assumption, and given Hull's specification of response probability $\left(R_{p}\right)$ as an ogival function of the superthreshold magnitude of effective reaction potential,1 Payne argued that additional reinforced practice and/or drive augmentation should not only reduce the drug-imposed deficit but perhaps even remove it altogether if the supplemental operations were extensive. This expectation, of course, presupposes that performance levels have reached the negatively accelerated portion of the $R_{p}$ function prior to drug administration, for it is only at that level that the supplemental operations should provide differential facilitation favoring the drug group. By the same token, there should be negligible differential effects at intermediate levels of the $R_{p}$ function, and augmentation of reaction potential at low levels should favor the placebo group. Payne's data supported the argument well by showing that $50 \%$ overtraining on a paired-associate verbal learning task was sufficient to bring about a substantial reduction of promethazine effects on relearning scores.

The foregoing conceptualization received impressive support from studies that employed other drugs, tasks, and organisms. For example, Singh (1964), Singh, Sharma, \& Manocha (1965), Singh \& Manocha (1966), and Ray \& Bivens (1966) showed that the magnitude of chlorpromazine effects on leverpressing in rats was significantly reduced by operations designed to increase habit strength. Moreover, two of these studies demonstrated mitigative contributions arising from increased drive (Singh, Sharma, \& Manocha, 1965; Singh \& Manocha, 1966), although there was some question in the former study as to whether increased drive had, in fact, achieved a proportional reduction of the chlorpromazine effect. On the negative side, neither Bindra \& Mendelson (1962) nor Mendelson \& Bindra (1962) were able to confirm expected reductions of drug effects.

Despite the balance of results favorable to Payne's hypothesis, the theoretical issue can scarcely be characterized as settled beyond dispute. The present study, therefore, sought to extend the scope of theoretical inquiry by assessing the single and joint effects of habit strength, drive, and drug variables on leverpressing to extinction. On the hypothesis outlined above, and given Hull's specification of responses to extinction ( $n$ ) as an increasing linear function of the magnitude of effective reaction potential, one should be able to show that, at low to moderate levels of the $n$ function, additional reinforced trials and/or increased deprivation should benefit drug and control groups essentially alike. SUBJECTS

Forty Long-Evans male hooded rats comprised the experimental sample. Weights ranged from 300 to $350 \mathrm{~g}$, and ages were approximately 80 days at the outset of the study.

\section{APPARATUS}

Gerbrands Model B rat chambers with pellet feeders and control units were used.

\section{PROCEDURE}

Experimental treatments were preceded by an adaptation period lasting 8 days. During the first 3 days, Ss were handled for $5 \mathrm{~min} /$ day. Additional handling and daily saline injections characterized the next 5 days, the last 3 of which included placement in the experimental chamber (bar removed) for $15 \mathrm{~min} /$ day.

Following the adaptation period, Ss were deprived of food for $48 \mathrm{~h}$ and given preliminary training in barpressing for Noyes Precision Food Pellet rewards. The shaping criterion was met when Ss emitted three responses within $5 \mathrm{~min}$, each response having been followed by food acquisition within 5 sec.

Upon completion of shaping, Ss were assigned without bias to the sampling plan shown in Table 1 , given the constraint that five Ss were allotted to each of the eight treatment combinations. The acquisition phase was conducted under $23-\mathrm{h}$ food deprivation. Extinction trials were initiated $24 \mathrm{~h}$ after completion of the acquisition phase. Experimental drive treatments were imposed by allowing half the $S$ s ad lib access to food for $21 \mathrm{~h}$ ( $3 \mathrm{~h}$ deprived), and the remaining Ss, ad lib access for $2 \mathrm{~h}$ ( $22 \mathrm{~h}$ deprived). Thirty minutes prior to extinction trials, half the $S$ s were injected with saline containing $1 \mathrm{mg}$ $\mathrm{CPZ} / \mathrm{kg}$ body weight, and the remaining $S s$ were injected with equivalent volumes of saline alone. $5 \mathrm{~min}$ was allowed, after which extinction was considered accomplished if further $\mathrm{Rs}$ were not forthcoming within $5 \mathrm{~min}$.

\section{RESULTS AND DISCUSSION}

An examination of shaping records showed that the treatment subclasses varied considerably in average times to first response and in average numbers of reinforced presses prior to arrival at the shaping criterion. These variations were seen as potential influences on response tendencies during extinction. Furthermore, an analysis of responses to extinction showed that the hypothesis of equal subclass variances was untenable. Accordingly, the values of these variables were converted to square roots and subjected to a covariance analysis in which the two shaping variables served as the covariates. The results are shown in Table 2, from which it is evident that resistance to extinction was significantly affected by all three experimental treatments. Computation of adjusted means showed that placebo Ss produced an average of 29.27 responses, while CPZ Ss During extinction, an initial period of

Table 1 Experimental Design

\begin{tabular}{|c|c|c|c|c|}
\hline \multirow[b]{3}{*}{$\begin{array}{l}\text { Acquisition } \\
\text { Conditions }\end{array}$} & \multicolumn{4}{|c|}{ Extinction Conditions } \\
\hline & \multicolumn{2}{|c|}{ Saline } & \multicolumn{2}{|c|}{ Chlorpromazine } \\
\hline & $\begin{array}{c}3-\mathrm{H} \\
\text { Deprivation }\end{array}$ & $\begin{array}{c}22-\mathrm{H} \\
\text { Deprivation }\end{array}$ & $\begin{array}{c}3-\mathrm{H} \\
\text { Devrivation }\end{array}$ & $\begin{array}{c}22-\mathrm{H} \\
\text { Deprivation }\end{array}$ \\
\hline $\begin{array}{l}5 \text { Reinforcements } \\
30 \text { Reinforcements }\end{array}$ & $\begin{array}{l}N=5 \\
N=5\end{array}$ & $\begin{array}{l}N=5 \\
N=5\end{array}$ & $\begin{array}{l}N=5 \\
N=5\end{array}$ & $\begin{array}{l}N=5 \\
N=5\end{array}$ \\
\hline
\end{tabular}


Table 2

Analysis of Adjusted $V$ ariances

\begin{tabular}{lccr}
\hline \multicolumn{1}{c}{ Source } & df & Adjusted \\
\hline Drug & 1 & $\mathbf{2 0 . 4 9 2 2}$ & F* \\
Motivation & 1 & 36.8305 & $\mathbf{8 . 9 2}$ \\
Reinforcement & 1 & $\mathbf{4 0 . 8 4 5 7}$ & $\mathbf{9 . 8 9}$ \\
DM & 1 & $\mathbf{3 . 9 8 8 9}$ & $<\mathbf{1 . 0 0}$ \\
DR & 1 & $\mathbf{7 . 4 2 7 9}$ & $\mathbf{1 . 8 0}$ \\
MR & 1 & $\mathbf{8 . 2 1 5 3}$ & $\mathbf{1 . 9 9}$ \\
DMR & 1 & $\mathbf{5 5 4 7}$ & $<\mathbf{1 . 0 0}$ \\
Error & $\mathbf{3 0} \dagger$ & $\mathbf{4 . 1 3}$ & \\
$\quad$ Total & $\mathbf{3 7} \dagger$ & & \\
\hline
\end{tabular}

*An $F$ of 4.17 is significant at the $5 \%$ level for $1 / 30 \mathrm{df}$.

tReduced by 2 df for regression coefficients.

$R_{y, x z}=.44, b_{1}=.81031, b_{2}=-.17771$.

produced an average of 15.84 responses. With respect to the drive effect, the 3-h Ss gave an average of 15.02 responses, while the 22 -h group posted an average of 32.14 responses. With respect to the reinforcement effect, the 5-reinforcement group produced an average of 13.66 responses, while the 30-reinforcement group gave an average of 33.93 responses. Thus, resistance to extinction was decreased by chlorpromazine and increased by augmented drive and habit strength. The increments of resistance were essentially alike for the saline and chlorpromazine groups, as shown by the negligible interaction variances recorded in Table 2 . The data were therefore consistent with theoretical expectation.

REFERENCES
BINDRA, D., \& MENDELSON, J. Interaction of habit strength and drug effects. Journal of Comparative \& Physiological Psychology, 1962, 55, 217-219.

HULL, C. L. Principles of behavior. New York: Appleton-Century-Crofts, 1943.

MENDELSON, J., \& BINDRA, D. Combination of drive and drug effects. Journal of Experimental Psychology,
$1962,63,505-509$.

PAYNE, R. B. An extension of Hullian theory to response decrements resulting from drugs. Journal of Experimental Psychology, 1958, 55, 342-346.

RAY, O. S., \& BIVENS, L. W. Performance as a function of drug, dose, and level of training. Psy chopharmacologia, 1966, 10, 103-109.

SINGH, S. D. Habit strength and drug effects. Journal of Comparative Physiological Psychology, 1964, 58 468-469.

SINGH, S. D., \& MANOCHA, S. N. The interaction of drug effects with drive level and habit strength. Psychopharmacologia, 1966, 9, 205-209.

SINGH, S. D., SHARMA, S., \& MANOCHA, $S$. N. Habit, drive, and drug effects. Psychological Studies, 1965, 10, 38-44.

SPENCE, K. W. Behavior theory and conditioning. New Haven: Yale University Press, 1956.

1. In Hull's (1943) theory, $R_{p}$ is assumed to be an S-shaped function of the extent to which $\bar{E}$ exceeds L (Postulate 12); but Spence (1956) later deduced this as a theorem from other assumptions (Postulates 4,10 , and 11 ), thereby increasing the parsimony of the theory. The authors are indebted to C. E. Noble for this reminder. 